\title{
Analysis and Remedy of Negativity Problem in Hybrid Stochastic Simulation Algorithm and its Application
}

\author{
Extended Abstract
}

\author{
Minghan Chen \\ Virginia Tech \\ Blacksburg, Virginia \\ cmhshirl@vt.edu
}

\author{
Yang Cao \\ Virginia Tech \\ Blacksburg, Virginia \\ ycao@cs.vt.edu
}

\begin{abstract}
The hybrid stochastic simulation algorithm, proposed by Haseltine and Rawlings, can significantly improve the simulation efficiency for multiscale biochemical networks. However, the population of some species might be driven negative under certain situations. This paper investigates the negativity problem of the hybrid method based on the second slow reaction firing time. Our analysis and tests on several models demonstrate that usually the error caused by negative populations is negligible compared with approximation errors of the method itself. But for systems involving nonlinear reactions or highly sensitive species, the system stability will be influenced and may lead to system failure. The proposed Zero-Reaction rule is recommended considering its efficiency and simplicity.
\end{abstract}

\section{CCS CONCEPTS}

- Theory of computation $\rightarrow$ Stochastic approximation; • Computing methodologies $\rightarrow$ Simulation evaluation; $\bullet$ Applied computing $\rightarrow$ Biological networks;

\section{KEYWORDS}

Hybrid stochastic algorithm; negative population; second slow reaction firing time

\section{ACM Reference Format:}

Minghan Chen and Yang Cao. 2018. Analysis and Remedy of Negativity Problem in Hybrid Stochastic Simulation Algorithm and its Application: Extended Abstract. In ACM-BCB'18: 9th ACM International Conference on Bioinformatics, Computational Biology and Health Informatics, August 29September 1, 2018, Washington, DC, USA. ACM, New York, NY, USA, 2 pages. https://doi.org/10.1145/3233547.3233683

\section{INTRODUCTION}

The stochastic simulation algorithm (SSA) [3], widely used in simulating stochastic effects in biochemical networks, is computationally intensive and inefficient for systems with fast reactions or large populations. As biological networks at single cell levels usually have large scale discrepancies in populations of species as well as

Permission to make digital or hard copies of part or all of this work for personal or classroom use is granted without fee provided that copies are not made or distributed for profit or commercial advantage and that copies bear this notice and the full citation on the first page. Copyrights for third-party components of this work must be honored. For all other uses, contact the owner/author(s).

ACM-BCB'18, August 29-September 1, 2018, Washington, DC, USA

(c) 2018 Copyright held by the owner/author(s).

ACM ISBN 978-1-4503-5794-4/18/08.

https://doi.org/10.1145/3233547.3233683 rate constants among different reactions, research is increasingly focused on hybrid methods targeting multiscale systems [1, 4]. Haseltine and Rawlings [4] proposed a hybrid method for general cases (hereafter referred to as the HR hybrid method), which modeled part of the system by continuous dynamics (ordinary differential equations (ODEs) or Langevin equations), while keeping the rest discrete. The idea of the HR hybrid method was further improved and extended to several hybrid methods and simulation tools $[5,6]$.

However, in the HR hybrid method framework, populations of some reactant species may become negative if they are involved in both deterministic and stochastic systems. Take system (1) as an example. If reaction rate constants satisfy $f_{1} \gg k_{c}$ and $b_{1} \gg k_{c}$, the system can be divided into a fast reaction group and a slow reaction group, containing the reversible and irreversible reactions, respectively.

$$
\mathrm{S}_{1} \underset{b_{1}}{\stackrel{f_{1}}{\rightleftharpoons}} \mathrm{S}_{2} \stackrel{k_{c}}{\longrightarrow} \mathrm{S}_{3} .
$$

Assume that this system has two $\mathrm{S}_{1}$ molecules at the beginning, and the system parameters are $f_{1}=1, b_{1}=9, k_{c}=0.01$. Then, compared with the slow system, the fast ODE system can be considered at equilibrium, which gives $x_{1}=1.8$ and $x_{2}=0.2$, where $x_{i}$ denotes the mean population of species $S_{i}$. Thus, when a slow reaction fires, $x_{2}$ is reduced to -0.8 . Negative populations may also appear in stochastic simulations of reaction-diffusion systems, especially when low-density species are distributed in a well-meshed space.

\section{SECOND SLOW REACTION FIRING TIME}

Our prior work [2] analyzed the accuracy of the HR hybrid method by studying the next slow reaction firing time (NSRFT). Since the negative population problem mostly emerges after a slow reaction, it is not enough to just study the first exit time. So, we further extend that work and study the second slow reaction firing time (SSRFT), which reflects the influence of a (possible) negative population on the firing of slow reactions. We use the same linear chain reaction network in Ref. [2] as a study example, shown below.

$$
\mathrm{S}_{1} \underset{b_{1}}{\stackrel{f_{1}}{\rightleftharpoons}} \mathrm{S}_{2} \underset{b_{2}}{\stackrel{f_{2}}{\rightleftharpoons}} \cdots \underset{b_{n-1}}{\stackrel{f_{n-1}}{\rightleftharpoons}} \mathrm{S}_{n} \stackrel{k_{c}}{\longrightarrow} \mathrm{S}_{n+1}
$$

A particle can exit the reversible chain system through $S_{n}$ with reaction rate $k_{c}$. In many applications, the reversible chain reactions can be considered as a fast subsystem and the irreversible reaction (exit to $S_{n+1}$ ) as a slow subsystem. With this partitioning strategy, if $x_{n}<1$, then $S_{n}$ will become negative when a slow reaction fires.

We proposed the following three strategies to handle the negativity problem. 
- Zero-Population: set all negative species' populations as zero whenever detected in the simulation.

- Zero-Reaction: set all reaction terms or propensities involving negative species as zero in corresponding subsystems until they become nonnegative.

- Zero-Time: when a species' population becomes negative, pause the system and run a separate virtual ODE system $\mathrm{G}_{f}^{\prime}$ that only contains reactions related to the negative species. When it becomes nonnegative, restart the original system with the new system state from $\mathrm{G}_{f}^{\prime}$.

We derived the formulas of SSRFT under the chemical master equation (CME), the HR hybrid method, and the three remedies (derivations omitted). For comparison, relative error is defined as

$$
e_{r}=\frac{\left|T_{c}-T_{h}\right|}{T_{c}},
$$

where $T_{c}$ and $T_{h}$ are the mean second slow reaction firing times of the CME and the HR hybrid method, respectively.

Fig. 1 illustrates the acceptable system parameter region $\left(e_{r}<\right.$ 0.01 ) for system sizes $n=2$ and $n=10$. In both cases, the SSRFT keeps the same pattern but with improved accuracy horizontally resulting from the negativity phenomenon and decreased accuracy vertically due to the accumulative method error. As the acceptable parameter region for larger linear chain systems gets smaller, the negativity problem becomes insignificant for the HR hybrid method. Since nonnegative situations occur much more frequently than negative situations, the three proposed remedies do not make a difference in the acceptable region of the HR hybrid method.

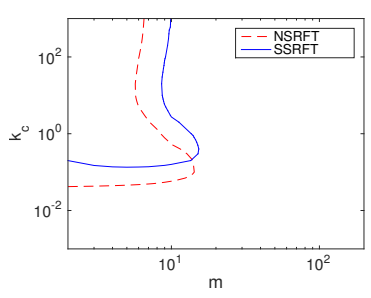

(a) $n=2$

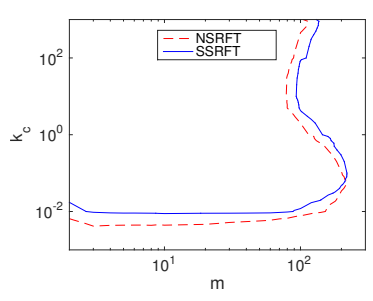

(b) $n=10$
Figure 1: Contour plot of the average relative error $e_{r}=0.01$ with different $\left(k_{c}, m\right)$ pairs. All system parameters are set to one. Acceptable parameter pairs for the HR hybrid method can be chosen from the bottom (small $k_{c}$ values) and right (large $m$ values) regions.

\section{NUMERICAL RESULTS ON A NONLINEAR SYSTEM}

The previous section have shown that the HR hybrid method works fine for linear systems. Here we examine the effect of negative values on a nonlinear system, shown below.

$$
\mathrm{S}_{1} \stackrel{f_{1}}{\underset{b_{1}}{\rightleftharpoons}} 2 \mathrm{~S}_{2} \stackrel{k_{c}}{\longrightarrow} \mathrm{S}_{3} \stackrel{k_{s}}{\longrightarrow} \mathrm{S}_{1} \text {. }
$$

Similarly, we partitioned the system into groups: the fast group containing the reversible reactions and the slow group containing the remaining reactions. For the bimolecular reaction $\mathrm{S}_{2}+\mathrm{S}_{2} \stackrel{k_{c}}{\longrightarrow} \mathrm{S}_{3}$, when $x_{2}<0$, the propensity $a_{b i}=k_{c} x_{2}\left(x_{2}-1\right)$ is positive which can potentially cause the reaction to fire and further decrease the value of $x_{2}$. Thus, the SSA system becomes unstable when $x_{2}<0$. Meanwhile, the ODE system becomes unstable when $\mathrm{S}_{2}$ gets smaller than $-\frac{f_{1}}{4 b_{1}}$ (one eigenvalue of the ODE system becomes positive).

In Fig. 2, our simulation results also proved that even if $x_{2}$ has less than a $1 \%$ probability to be negative, the system suffers significant error and breaks down after a certain amount of simulation time when using the HR hybrid method. Only the Zero-Reaction rule still works and generates similar results to the SSA. The Zero-Time rule failed because the separate $\mathrm{G}_{f}^{\prime}$ system is unstable.

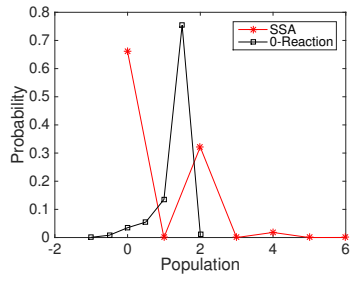

(a) $\mathrm{S}_{2}$

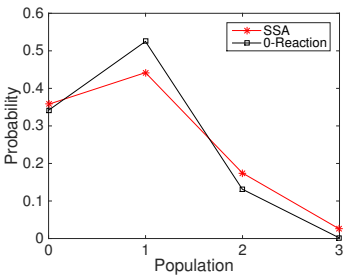

(b) $\mathrm{S}_{3}$
Figure 2: Final distributions of species $S_{2}$ and $S_{3}$ from the SSA, the Zero-Reaction remedy based on $10 \mathrm{k}$ simulations. The parameters for the nonlinear system are $f_{1}=b_{1}=k_{c}=$ $k_{s}=1$. The initial condition is $m_{1}=3, m_{2}=0$.

\section{SUMMARY AND CONCLUSIONS}

This paper studies the negativity problem of the HR hybrid stochastic simulation algorithm based on the second slow reaction firing time. We conclude that the negativity phenomenon does not influence the biochemical network unless the negative species are involved in nonlinear reactions that generate positive reacting terms or propensities. The proposed Zero-Reaction strategy can handle nonlinear systems with minimal computational cost.

\section{ACKNOWLEDGMENTS}

This work was partially supported by the National Science Foundation under awards CCF-0953590, CCF-1526666, and MCB-1613741.

\section{REFERENCES}

[1] Yang Cao, Daniel T. Gillespie, and Linda R. Petzold. 2005. The slow-scale stochastic simulation algorithm. F. Chem. Phys. 122, 1 (2005), 014116.

[2] Minghan Chen, Shuo Wang, and Yang Cao. 2018. Accuracy analysis of hybrid stochastic simulation algorithm on linear chain reaction systems. (2018). To appear, Bulletin of Math Biology.

[3] Daniel T. Gillespie. 1976. A general method for numerically simulating the stochastic time evolution of coupled chemical reactions. f. Comput. Phys. 22, 4 (1976), 403-434.

[4] Eric L. Haseltine and James B. Rawlings. 2002. Approximate simulation of coupled fast and slow reactions for stochastic chemical kinetics. F. Chem. Phys. 117, 15 (2002), 6959-6969.

[5] Paola Lecca, Fabio Bagagiolo, and Marina Scarpa. 2017. Hybrid deterministic/stochastic simulation of complex biochemical systems. Mol. BioSyst. 13 (2017), 2672-2686.

[6] Zhen Liu, Yang Pu, Fei Li, Clifford A. Shaffer, Stefan Hoops, John J. Tyson, and Yang Cao. 2012. Hybrid modeling and simulation of stochastic effects on progression through the eukaryotic cell cycle. F. Chem. Phys. 136, 3 (2012), 034105. 